\title{
Suppression of Anderson Localization in Disordered Metamaterials
}

\author{
Ara A. Asatryan, ${ }^{1}$ Lindsay C. Botten, ${ }^{1}$ Michael A. Byrne, ${ }^{1}$ Valentin D. Freilikher, ${ }^{2}$ Sergey A. Gredeskul, ${ }^{3,4}$ \\ Ilya V. Shadrivov, ${ }^{4}$ Ross C. McPhedran, ${ }^{5}$ and Yuri S. Kivshar ${ }^{4}$ \\ ${ }^{1}$ Department of Mathematical Sciences and Center for Ultrahigh-bandwidth Devices for Optical Systems (CUDOS), \\ University of Technology, Sydney, NSW 2007, Australia \\ ${ }^{2}$ Department of Physics, Bar-Ilan University, Raman-Gan, 52900, Israel \\ ${ }^{3}$ Department of Physics, Ben Gurion University of the Negev, Beer Sheva, 84105, Israel \\ ${ }^{4}$ Nonlinear Physics Center and Center for Ultrahigh-bandwidth Devices for Optical Systems (CUDOS), \\ Australian National University, Canberra, ACT 0200, Australia \\ ${ }^{5}$ School of Physics and Center for Ultrahigh-bandwidth Devices for Optical Systems (CUDOS), University of Sydney, \\ Sydney, NSW 2006, Australia \\ (Received 23 May 2007; revised manuscript received 4 October 2007; published 9 November 2007)
}

\begin{abstract}
We study wave propagation in mixed, 1D disordered stacks of alternating right- and left-handed layers and reveal that the introduction of metamaterials substantially suppresses Anderson localization. At long wavelengths, the localization length in mixed stacks is orders of magnitude larger than for normal structures, proportional to the sixth power of the wavelength, in contrast to the usual quadratic wavelength dependence of normal systems. Suppression of localization is also exemplified in long-wavelength resonances which largely disappear when left-handed materials are introduced.
\end{abstract}

DOI: 10.1103/PhysRevLett.99.193902

PACS numbers: 42.25.Dd, 42.25.Fx, 72.15.Rn

Anderson localization is amongst the most fascinating and universal phenomena in the physics of disordered systems $[1,2]$. Despite the many rigorous results for 1D disordered systems $[3,4]$, the study of classical wave localization [5] still reveals many novel and fundamental properties, e.g., those associated with absorption [6] and gain $[7,8]$. In this Letter, we consider wave propagation in disordered, mixed metamaterials and reveal yet another fascinating feature of Anderson localization.

Left-handed metamaterials [9] have received considerable attention recently, given their unique ability to resolve images beyond the diffraction limit $[9,10]$ and their capacity to cloak objects [11]. To date, most studies consider ideal systems [12] and do not address the effects of disorder, although Gorkunov et al. [13] demonstrated that weak microscopic disorder may lead to substantial suppression of wave propagation using magnetic metamaterials, while Dong and Zhang [14] considered localization due to thickness disorder in alternating layers of normal and metamaterials that were not impedance matched.

From simple arguments, it is clear that the mutual influence of disorder and negative refraction may lead to nontrivial effects, either through evanescent wave amplification or the modification of wave phase due to negative phase velocity. Here, however, there are no evanescent waves and so the fascinating, and perhaps counterintuitive, effects that we observe are attributable to the impact of disorder on the interference in stacks of materials in which the phase velocity alternates.

A key characteristic of Anderson localization is the localization length $l$. In normal, disordered systems comprising right-handed $(\mathrm{RH})$ media, $l$ is proportional to the square of the wavelength $\lambda$ in the long-wavelength limit, tends to a constant in the short-wavelength regime, and oscillates in the intermediate wavelength region $[2,15-18]$. Here, we consider 1D disordered systems of mixed (M) stacks composed of $N$ layers of alternating RH and lefthanded (LH) materials (see Fig. 1), and show that the matched combination of LH- and RH-layers causes not only an increase in $l$ by several orders of magnitude, but also changes dramatically its functional dependence: instead of the well-known, long-wavelength asymptotic, $l \propto$ $\lambda^{2}$, for disordered homogeneous (H) stacks (i.e., comprising purely RH or purely LH layers), we have $l \propto \lambda^{6}$ for the $M$ stack in the long wave limit.

We consider a disordered structure of $N$ (even) layers (Fig. 1). In layer $j$, the permeability and permittivity are $\mu_{j}= \pm 1$ and $\varepsilon_{j}= \pm\left(1+\delta_{j} \pm i \sigma\right)^{2}$, respectively, for the RH and LH layers, and we consider both lossless $(\sigma=0)$ and absorbing $(0<\sigma \ll 1)$ structures. The refractive index fluctuations $\delta_{j}$ are independent random variables distributed uniformly on $[-Q, Q]$. With this, the refractive index and the impedance of each slab, relative to the

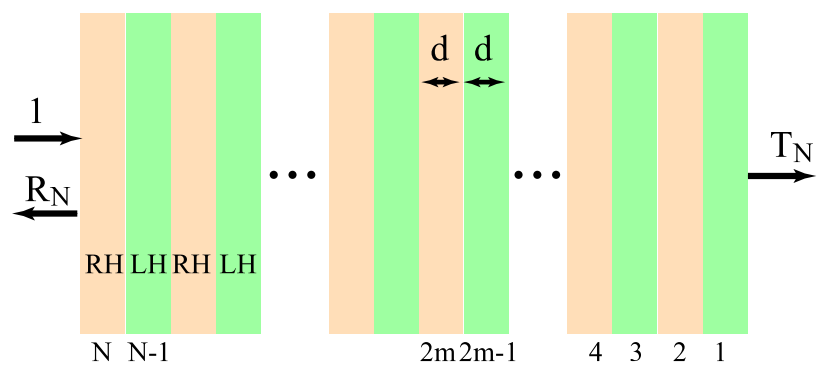

FIG. 1 (color online). Geometry of a disordered metamaterial structure. 
background (free space), are $n_{j}=\sqrt{\varepsilon} \bar{\mu}= \pm\left(1+\delta_{j} \pm i \sigma\right)$ and $Z_{j}=\sqrt{\mu_{j} / \varepsilon_{j}}=1 /\left(1+\delta_{j} \pm i \sigma\right)$ with the choice of sign corresponding to RH and LH slabs, respectively. We assume that all layers have identical thickness $d$ and we make use of dimensionless variables, measuring all lengths in units of $d$. The total length of the stack is thus $N$, the number of layers.

First, we study lossless systems $(\sigma=0)$ and notice that when our structure is periodic $(Q=0)$, it is transparent at all frequencies since there is no impedance mismatch at any boundary. That is, there are no forbidden bands in the spectrum of the structure and, therefore, disorder in the refractive index of the layers provides the only physical mechanism for wave localization. Interestingly, the introduction of thickness disorder to such a refractively unperturbed system conserves its complete transparency at all frequencies, irrespective of the strength of the disorder, a consequence of layer impedance matching.

In this Letter, we study the properties of the dimensionless localization length $l$, defined as the reciprocal of the Lyapunov exponent $\gamma[1,3]$,

$$
\gamma \equiv l^{-1}=\lim _{N \rightarrow \infty} \frac{\xi_{N}}{N}=-\lim _{N \rightarrow \infty} \frac{\ln \left|T_{N}\right|}{N}
$$

where $\xi_{N}$ is the natural logarithm of the magnitude of a "zero-current" (fixed at the input) solution of the corresponding dynamical equations, and $T_{N}$ is the transmission amplitude of a random stack of $N$ layers. Because of the self-averaging of the Lyapunov exponent, the numerator in the final term of Eq. (1) can be replaced by its ensemble average value. We thus introduce two lengths,

$$
l_{\xi}(N)=\frac{N}{\xi_{N}}, \quad \text { and } \quad l_{T}(N)=-\frac{N}{\left\langle\ln \left|T_{N}\right|\right\rangle} .
$$

The former is calculated for any single realization, while the latter is computed by averaging its denominator over many random configurations. For a sufficiently long stack, both lengths almost coincide and practically do not change with further increases in system length. If these length scales are much smaller than the total length of the structure, i.e., $l_{\xi}, l_{T} \ll N$, then either can be used to characterize the genuine localization length.

To calculate $l_{\xi}$ and $l_{T}$, we use recurrence relations derived from the transfer matrix method [19]. For any random realization, we find $T_{N}$ from the recurrence relations for the stack transmission and reflection amplitudes,

$$
T_{j}=\frac{T_{j-1} t_{j}}{1-R_{j-1} r_{j}}, \quad R_{j}=r_{j}+\frac{R_{j-1} t_{j}^{2}}{1-R_{j-1} r_{j}},
$$

and then calculate $l_{T}(N)$ (2). Here, $R_{j}$ and $T_{j}$ denote reflection and transmission amplitudes of a $j$ layer stack, enumerated from $j=1$ at the rear through to $j=N$ at the front, and with layer $j$ characterized by reflection and transmission amplitudes $r_{j}$ and $t_{j}$ which are functions of $n_{j}, Z_{j}$, and the phase change across the layer, $\phi_{j}$.

To calculate $l_{\xi}$, it is convenient to choose pairs of adjacent LH and RH layers as the basic building block. Then,

$$
\frac{1}{l_{\xi}}=\frac{1}{N} \sum_{m=1}^{N / 2} \ln \left|\mathcal{T}_{22}^{(m)}+\zeta_{m-1} \mathcal{T}_{21}^{(m)}\right|,
$$

where $\mathcal{T}_{i k}^{(m)}$ denote elements of the transfer matrix [17,19] for layer pair $m$, expressed in terms of $Z_{j}, n_{j}$, and $\phi_{j}$, while the dynamical variable $\zeta_{m}$ satisfies

$$
\zeta_{m}=\frac{\mathcal{T}_{22}^{(m) *} \zeta_{m-1}+\mathcal{T}_{21}^{(m) *}}{\mathcal{T}_{22}^{(m)}+\mathcal{T}_{21}^{(m)} \zeta_{m-1}}, \quad \zeta_{0}=1
$$

A similar procedure may also be used to calculate a single realization of $\ln \left|T_{N}\right|$, used in the calculation of $l_{T}$. Accordingly, these two approaches complement each other well and, as should be expected, the results for $l_{\xi}$ and $l_{T}$ agree very closely and coincide in the region where both represent the genuine localization length.

Our results, summarized in Figs. 2 and 3, reveal a number of interesting and unexpected features. These show the wavelength dependence of $l_{\xi}$ and $l_{T}$ over various random configurations for the disorder $Q=0.25$. The solid line in Fig. 2 corresponds to $l_{\xi}$ for propagation in a $M$ stack for a single realization of $N=10^{9}$ layers, while the dashed line is for the corresponding $H$-stack. Figure 3 depicts $l_{T}$ for three different $M$ stacks of $N=10^{7}, 10^{5}, 10^{3}$ layers (from top to bottom) and the same quantity for a $H$ stack of $N=10^{4}$ layers. In all cases, $\left\langle\ln \left|T_{N}\right|\right\rangle$, in the denominator of Eq. (2), is averaged over $10^{4}$ realizations,

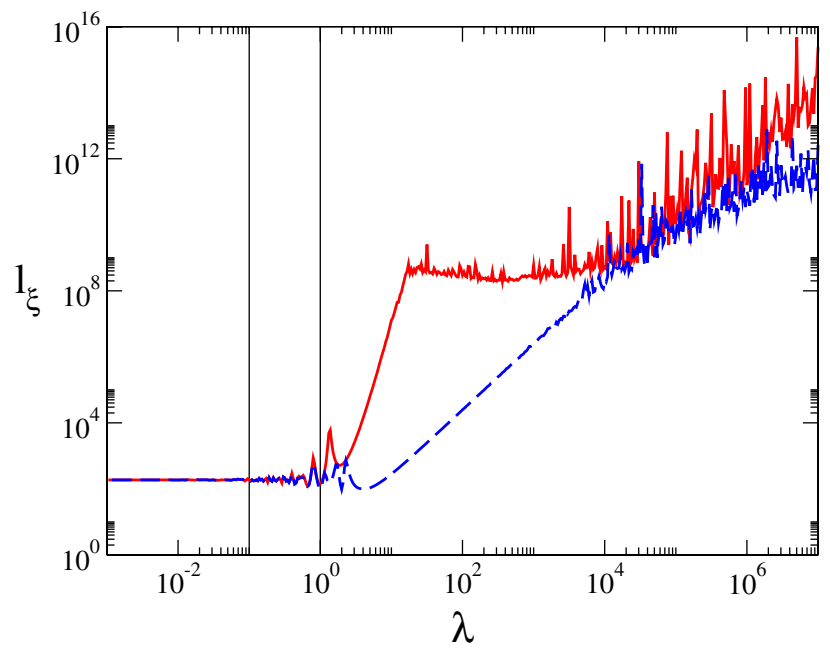

FIG. 2 (color online). Characteristic length $l_{\xi}$ vs wavelength $\lambda$ for $Q=0.25$ and $N=10^{9}$ layers; solid line is for the $M$ stack, while the dashed line is for the corresponding (normal) $H$ stack. The vertical lines at $\lambda=0.1,1$ delimit the short-, intermediate-, and long-wavelength regimes. 


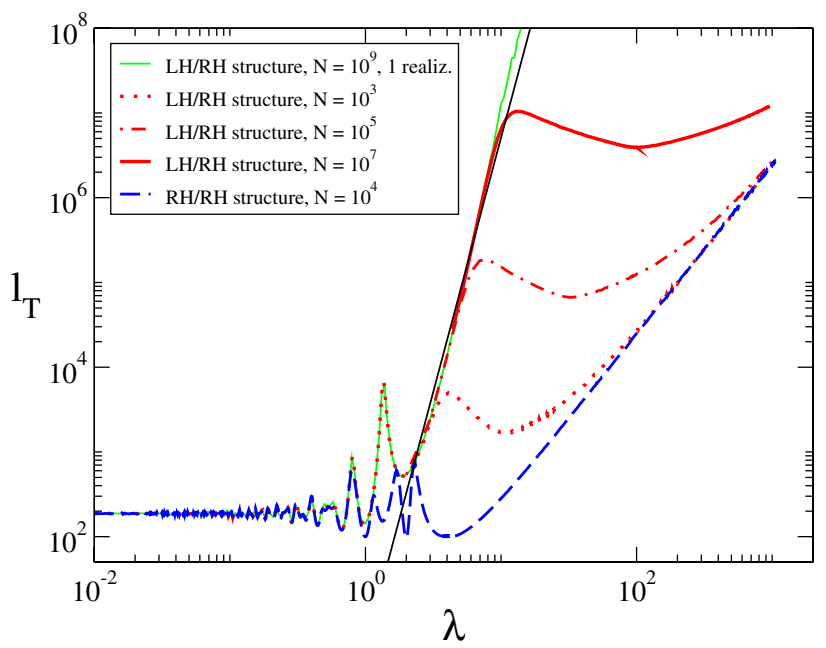

FIG. 3 (color online). Characteristic length $l_{T}$ vs $\lambda$ for $Q=$ 0.25 . The inset shows the legend for $M$ - and $H$ stack spectra. The fitted straight line is $l_{T} \approx 5.1 \lambda^{6}(6)$. To facilitate comparison of the single realization (Fig. 2) and ensemble averaged calculations, $l_{\xi}$ for the $M$ stack of Fig. 2 is also plotted on this graph.

sufficient to achieve graphical accuracy. As can be seen from Figs. 2 and 3, for $\lambda \lesssim 10$, both $l_{\xi}$ and $l_{T}$ are smooth functions (the former due to self-averaging) which are (a) essentially identical, (b) much smaller than the length of the sample $N$, and (c) independent of $N$. These thus represent the genuine localization length $l$, with this being further exemplified in Fig. 3.

From Figs. 2 and 3, we see that, in the short-wavelength regime $(\lambda \ll 1)$, the localization length of the $M$ stack remains constant [15-18], while for $0.5 \lesssim \lambda \lesssim 1$, it exhibits oscillations similar to that seen in disordered $H$ stacks [18]. However, for long-wavelengths, $\lambda \gtrsim 2$, the situation is markedly different. Instead of the well established asymptotic form $l \propto \lambda^{2}$, applicable to disordered $H$ stacks, the localization length of a $M$ stack grows much more rapidly, with simulations for a range of values of the disorder parameter $Q(0.01 \leq Q \leq 0.3)$ revealing that

$$
l_{\xi}, l_{T} \propto \lambda^{6} .
$$

Thus, the inclusion of left-handed metamaterial layers in the disordered stack substantially suppresses Anderson localization in the long-wavelength limit - the essential difference between $M$ and $H$ stacks being the much weaker interference in $M$ stacks, attributable to a lack of phase accumulation over the sample, due to the cancellation of phase across alternating $\mathrm{LH}$ and RH layers.

For $\lambda \gtrsim 10, l_{\xi}$ and $l_{T}$ are quite different, with $l_{\xi}$ exhibiting giant, irregular oscillations (Fig. 2) that appear in all realizations. At such wavelengths, the stack is not sufficiently long for self-averaging, needed for $l_{\xi}$ to attain its (nonrandom) limit. In contrast, the length $l_{T}$ is smooth even for $\lambda \geqslant 10$, due to ensemble averaging (2). Here, however, $l_{T}$ does not represent the genuine localization length since it is larger than $N$, the total length of the system. Nevertheless, in this ballistic transport regime, $l_{T}$ is still a physically meaningful quantity. Here, the transmittance $\left|T_{N}\right|^{2}$ is close to unity, exhibits strong relative fluctuations in $1-\left|T_{N}\right|^{2}$, and has an average value [2,20] of $\left\langle\left|T_{N}\right|^{2}\right\rangle=1-2 N / l_{\text {bal }}$, where the ballistic length $l_{\text {bal }}$ is much larger than $N$.

For $H$ stacks, it follows that $l_{T}=-N /\left(\left\langle\ln \left|T_{N}\right|\right\rangle\right) \approx l_{\text {bal }}$ (2), coinciding with the same length that occurs in the localization limit, $l_{T}(\ll N)$. That is, the transmission properties of a normal stack, in both the localized and ballistic regimes, are characterized by a single length scale, proportional to $\lambda^{2}$. In contrast, and somewhat surprisingly, the long-wavelength properties of mixed stacks are described by two different characteristic lengths: the localization length, proportional to $\lambda^{6}$, and the ballistic length, proportional to $\lambda^{2}$ (See Fig. 3).

In addition to the differences in the behavior of the localization length exhibited by homogeneous and mixed media, another discriminating characteristic is their resonance properties. Figure 4 presents a single realization of $\left|T_{N}\right|^{2}$ as a function of $\lambda$ for an $M$ stack (dashed line) of $N=10^{3}$ layers, and for the corresponding $H$ stack (solid line). From this, we see that the disordered $H$ stack exhibits resonances over the entire spectrum, while there are no resonances for the $M$ stack for $\lambda \geqslant 4$. While this, at first, may be unexpected, it is just a further manifestation of the lack of phase accumulation over the length of the system: for this realization, the accumulated wave phase in the mixed stack did not exceed $\pi / 2$. This behavior is replicated over all realizations and is consistent with our earlier observation that, for mixed media, much longer stacks are required to cause localization.

We have also studied localization in $M$ stacks that include absorption, present in all real metamaterials. Here, the attenuation of the field is attributable to both

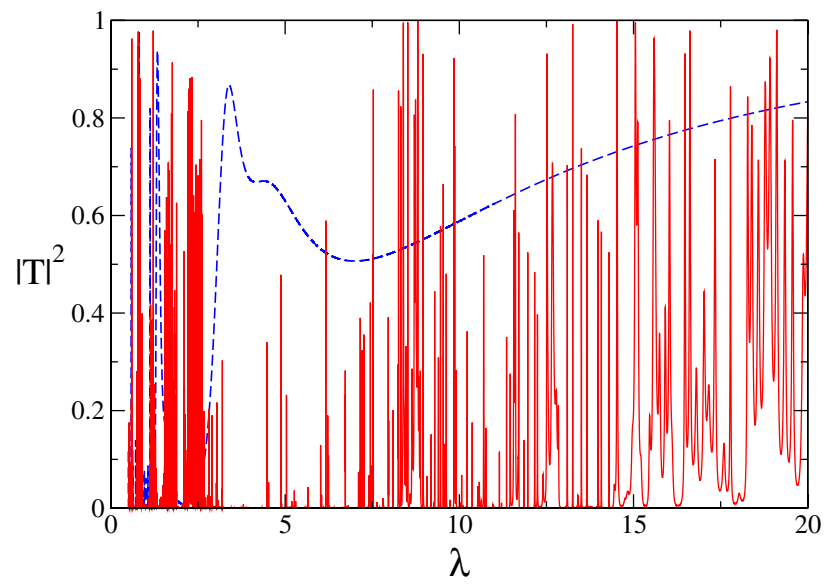

FIG. 4 (color online). Transmittance $|T|^{2}$ vs $\lambda$ for a single realization $\left(Q=0.25, N=10^{3}\right)$. Solid: normal $H$ stack, dotted: $M$ stack. 


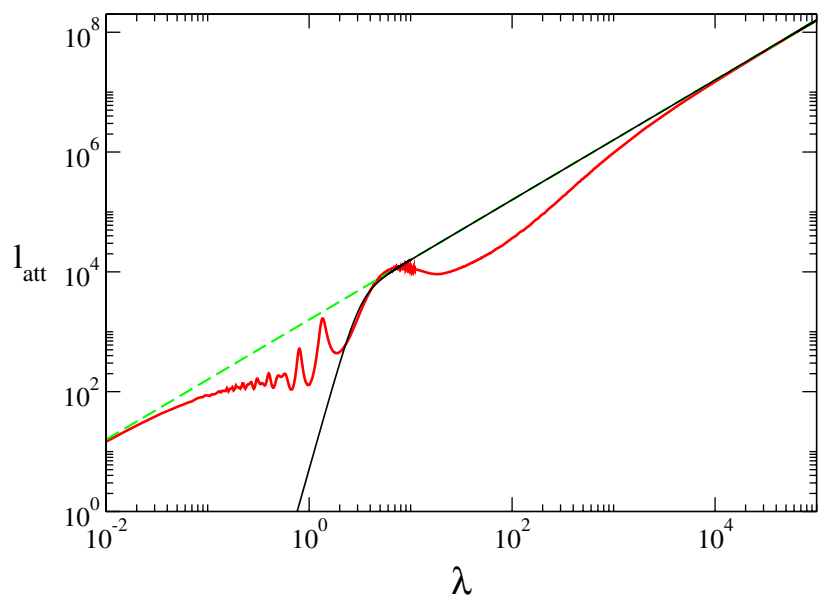

FIG. 5 (color online). Attenuation length $l_{\text {att }}$ vs $\lambda$ for a $M$ stack with $Q=0.25, \sigma=10^{-4}, N=10^{4}$. Solid (red) line: numerical results; dashed (green) line: $l_{\mathrm{abs}}$; solid black line: $l_{\mathrm{att}}$, where $l_{\mathrm{att}}^{-1} \approx l_{T}^{-1}+l_{\mathrm{abs}}^{-1}$.

Anderson localization and absorption, and so $l_{\text {att }}(N)=$ $-N /\left\langle\ln \left|T_{N}\right|\right\rangle$ has the meaning of an attenuation length. Figure 5 plots $l_{\text {att }}$ for an $M$ stack with $N=10^{4}$ and $\sigma=$ 0.0001 (solid line), and the absorption length $l_{\text {abs }}=$ $\lambda /(2 \pi \sigma)$ (dashed line). Clearly, absorption dominates in the short-wavelength region, $\lambda \leqslant 0.01$, and also in the long-wavelength region, $\lambda \geqslant 1000$, while for intermediate wavelengths, $0.1 \lesssim \lambda \lesssim 10$, the main contribution to the attenuation is localization. The only difference is that due to absorption, with $l_{\text {att }}$ for the lossy sample having fewer oscillations in the resonance region than $l_{T}$ for the lossless case. These effects combine through $l_{\mathrm{att}}^{-1} \approx l_{T}^{-1}+l_{\mathrm{abs}}^{-1}$, with the solid black curve of Fig. 5 displaying $l_{\text {att }}$, in which $l_{T}=$ $c \lambda^{6}(c \approx 5.1)$ is taken from the equivalent lossless system. Clearly, this is an excellent fit to the simulations in both the localization and long-wavelength regimes.

In conclusion, we have shown that in stratified media with alternating layers of right- and left-handed materials, the localization properties differ dramatically from those exhibited by conventional, disordered materials. In particular, at long wavelengths, the localization length of mixed, metamaterial stacks is proportional to the sixth power of the wavelength, a result which has been neither predicted nor observed in conventional 1D disordered systems. In contrast to normal, disordered materials, the characteristic ballistic and localization lengths of mixed stacks differ substantially in the weak scattering limit, with the length of the mixed stack needed to realize transmission resonances being much greater than for the corresponding normal sample. This shows that left-handed metamaterials can substantially suppress Anderson localization in 1D disordered systems.

This work was supported by the Australian Research Council under its Centres of Excellence and Discovery Grants programs, and the Israel Science Foundation (Grant No. 944/05). Yu. K., A. A., and L. B. thank C. Soukoulis and P. Sheng for discussions. V.F. and S. G., respectively, thank the University of Technology Sydney, and the Nonlinear Physics Center, Australian National University, for hospitality. We acknowledge ac3 and APAC for access to high performance computing facilities.

[1] I. M. Lifshitz, S. A. Gredeskul, and L. A. Pastur, Introduction to the Theory of Disordered Systems (Wiley, New York, 1989).

[2] P. Sheng, Introduction to Wave Scattering, Localization, and Mesoscopic Phenomena (Academic, New York, 1995).

[3] V.D. Freilikher and S. A. Gredeskul, Progress in Optics, edited by E. Wolf (Elsevier Science, Amsterdam, 1992), Chap. III, Vol. XXX, p. 137.

[4] S. A. Gredeskul, A. V. Marchenko, and L. A. Pastur, in Surveys in Applied Mathematics (Plenum, New York, 1995), Vol. 2, p. 63.

[5] K. Yu. Bliokh, Yu. P. Bliokh, and V. D. Freilikher, J. Opt. Soc. Am. B 21, 113 (2004).

[6] V. D. Freilikher, M. Pustilnik, and I. Yurkevich, Phys. Rev. Lett. 73, 810 (1994).

[7] J.C.J. Paasschens, T. Sh. Misirpashaev, and C. W. J. Beenakker, Phys. Rev. B 54, 11887 (1996).

[8] A. A. Asatryan et al., Phys. Rev. B 57, 13535 (1998).

[9] V. G. Veselago, Sov. Phys. Usp. 10, 509 (1968).

[10] J. B. Pendry, Phys. Rev. Lett. 85, 3966 (2000).

[11] D. Schurig et al., Science 314, 977 (2006).

[12] J. Li, L. Zhou, C. T. Chan, and P. Sheng, Phys. Rev. Lett. 90, 083901 (2003).

[13] M. V. Gorkunov, S. A. Gredeskul, I. V. Shadrivov, and Yu. S. Kivshar, Phys. Rev. E 73, 056605 (2006).

[14] Y. Dong and Z. Zhang, Phys. Lett. A 359, 542 (2006).

[15] S. John, H. Sompolinsky, and M. J. Stephen, Phys. Rev. B 27, 5592 (1983).

[16] M. Ya. Azbel, Phys. Rev. B 28, 4106 (1983).

[17] V. Baluni and J. Willemsen, Phys. Rev. A 31, 3358 (1985).

[18] C. Martijn de Sterke and R. C. McPhedran, Phys. Rev. B 47, 7780 (1993).

[19] M. Born and E. Wolf, Principles of Optics (Cambridge University Press, Cambridge, 2002).

[20] S. M. Rytov, Yu. A. Kravtsov, and V. I. Tatarskii, Principles of Statistical Radiophysics (Springer, Berlin, 1987). 